\title{
Situational Motivation and Perceived Intensity: Their Interaction in Predicting Changes in Positive Affect from Physical Activity
}

\author{
Eva Guérin and Michelle S. Fortier \\ School of Human Kinetics, University of Ottawa, 125 University Private Montpetit Hall, Ottawa, ON, Canada K1N 6N5 \\ Correspondence should be addressed to Eva Guérin, eguer016@uottawa.ca
}

Received 8 December 2011; Revised 20 April 2012; Accepted 28 April 2012

Academic Editor: Pedro J. Teixeira

Copyright ( $) 2012$ E. Guérin and M. S. Fortier. This is an open access article distributed under the Creative Commons Attribution License, which permits unrestricted use, distribution, and reproduction in any medium, provided the original work is properly cited.

\begin{abstract}
There is evidence that affective experiences surrounding physical activity can contribute to the proper self-regulation of an active lifestyle. Motivation toward physical activity, as portrayed by self-determination theory, has been linked to positive affect, as has the intensity of physical activity, especially of a preferred nature. The purpose of this experimental study was to examine the interaction between situational motivation and intensity [i.e., ratings of perceived exertion (RPE)] in predicting changes in positive affect following an acute bout of preferred physical activity, namely, running. Fourty-one female runners engaged in a 30-minute self-paced treadmill run in a laboratory context. Situational motivation for running, pre- and post-running positive affect, and RPE were assessed via validated self-report questionnaires. Hierarchical regression analyses revealed a significant interaction effect between RPE and introjection $(P<.05)$ but not between RPE and identified regulation or intrinsic motivation. At low levels of introjection, the influence of RPE on the change in positive affect was considerable, with higher RPE ratings being associated with greater increases in positive affect. The implications of the findings in light of SDT principles as well as the potential contingencies between the regulations and RPE in predicting positive affect among women are discussed.
\end{abstract}

\section{Introduction}

Physical activity (PA) has been identified as a hallmark contributor to individuals' quality of life [1], most notably among women [2]. Indeed, several research endeavours have converged on trying to understand the principal determinants of PA, and in particular, those factors that can be associated with individuals' volitional control of this specific behaviour. One factor that has been of mounting interest to behavioural scientists is that of affect and/or mood, which is defined as the quality of a subjective feeling state consisting of elements of valence (i.e., good/bad) and activation (i.e., high/low) [3]. Notably, studies have revealed associations between engaging in an acute bout of PA and immediate increases in positive affect [4]. In turn, basic affective reactions that are tied to a moderate intensity PA session (i.e., increases in positive affect) have been shown to predict futures bouts of PA six and twelve months later [5]. Indeed, repeated positive affective experiences associated with PA may sustain PA motivation over time and this can facilitate longterm PA participation $[6,7]$. It follows then that studying positive affect in the context of specific PA sessions is an outcome worthy of investigation in its own right, and this within broader attempts to understand how to optimally predict sustainable patterns of PA behaviour for successful weight management and greater well-being.

However, experts have highlighted that not all individuals who participate in PA achieve more positive affective states and ensuing increments in general well-being [8]. Indeed, results have not been entirely consistent as some participants have witnessed no change or a worsening of their affect with exercise [9]. This may have long-term implications in terms of sustaining adequate amounts of PA as well as levels of well-being. This has led to researchers' attempts to isolate the conditions under which the specific affective benefits of exercise might be maximized or conversely, thwarted.

In particular, one line of research has focused on the intensity of PA and its relationship with exercise-related 
affect. Meta-analytic findings have revealed that the effect of acute PA on positive-activated affect may be stronger at low-to-moderate intensities $[4,10]$. In past studies, some researchers have attempted to uncover a "threshold of intensity" for the affective benefits of exercise, yet there is insufficient empirical evidence for an inverted $U$ shape doseresponse relationship [11]. From a more person-centered view, several researchers now argue that preferred/self-selected intensity might lead to stronger benefits in positive affect than prescribed PA intensities $[12,13]$. Overall, the evidence is inconclusive as there appears to be significant interindividual variability regarding the benchmark intensity level that is most conducive to increments in positive affect.

Another line of work that has specifically targeted such individual-based differences has also addressed a call for more theoretical research on possible psychological mechanisms underlying the PA-positive affect relationship [14, 15]. In particular, it has been suggested and recently emphasized that individuals' motivational styles toward PA may supply a missing and understudied link [16]. Self-determination theory (SDT), one recognized and well-supported motivational theory, distinguishes between two general types of motivation: self-determined motivation and controlled motivation (or regulation) [17]. Deci and Ryan [17] also classified motivation into several types of behavioural regulations that fall within these two broader categories. Overall, self-determined motivation is rooted in feelings of satisfaction, choice and volition. It is characterized as enjoying an activity for its own sake (i.e., intrinsic motivation) and/or assigning it value and personal importance (i.e., identified regulation). Controlled or non-self-determined motivation can be defined as engaging in an activity, such as exercise, so as to avoid self-inflicted shame or guilt, or to gain a personal reward, namely pride (introjected regulation) [18]. Controlled motivation can also be characterized by being motivated according to external demands (e.g., to obtain a reward; external regulation) [18]. Self-determination theory also postulates another type of motivation, amotivation, which was deemed less relevant in the present study with active participants as it is defined as lacking the intention to act [17].

Self-determination, namely, higher levels of intrinsic and identified styles of motivation, has been associated with healthy intentions to engage in health-promoting behaviours such as PA [19]. In women, identified regulation has also stood out as a predictor of the intensity of PA engagement [20]. Higher levels of these self-determined regulations have also been positively associated with desirable psychological variables including PA enjoyment [21] and post-PA positive affect [22], as well as a battery of well-being indicators such as greater self-esteem and lower depression and state anxiety levels [23].

On the other hand and under the more controlling classifications, introjected regulation is considered less favourable than self-determined motivational styles [18]. Indeed research has revealed an association between introjected motivation for PA and maladaptive outcomes that include poorer life satisfaction [24] and exercise-dependence symptoms such as strenuous PA [25]. Similarly higher scores on external motivation have been linked to self-esteem issues and higher levels of negative affect [26].

Although motivation toward PA and the perceived intensity of PA have seemingly been construed as independent influences, it could be that they exert an interaction effect on PA-induced affective changes. Such an interplay would be consistent with the expert-noted complexity of underlying mechanisms of the PA-affect (and well-being) relationship as well as new evidence regarding the relevance of motivational styles in predicting changes in affect following PA at selfselected intensities $[15,16]$. Especially with introjected regulation, which theory proposes should not be directly linked to well-being (and affect), perceived intensity may exert a particular contributing influence [27]. While other research, albeit scant, has underscored the possible interplay between self-selected PA intensity and psychological factors such as personality [28] and self-efficacy [29], to our knowledge no empirical research has properly entertained the interaction with motivation. Indeed, an interplay between perceived intensity (self-selected) and motivation styles in predicting changes in affect with acute PA could reveal a sustainable mechanism for long-term PA and well-being that lies within individuals' volitional regulation and that also capitalizes on the physical properties of exercise.

Therefore, the purpose of this controlled laboratory study was exploratory and was to examine whether there was an interaction between SDT's motivational regulations to engage in a running activity and Ratings of Perceived Exertion (RPE; self-selected intensity) in predicting pre-to post-PA (i.e., running) changes in positive affect. The three types of motivation were targeted given evidence cited above as well as recently revealed associations between motivation types and intensity preferences. Lastly, this study was conducted with active women given that the link between PA and affect may be particularly evident in active individuals experienced with PA $[30,31]$ as well as among women [32, 33]. This would allow for a "model" sample to highlight the above underlying theoretical relationships; in addition, this would contribute to an important need to better understand how to develop successful of PA intervention strategies for women [34].

\section{Methods}

2.1. Participants. Fourty-one healthy and active women (i.e., $>20$ minutes of moderate to vigorous PA three times per week) with an average age of $40.98(\mathrm{SD}=4.93)$ participated in this study. The Godin Leisure Time Exercise Questionnaire [35], a common and validated self-report measure of PA levels, was administered as a screening measure and the mean score for this sample was high at 59.71. The participants also had a low-average BMI of 22.47. To minimize confounding influences in the experimental protocol, it was necessary that participants self-report running as their preferred or most frequent exercise modality [36]. In their leisure time, the women reported running at a mean RPE of 13.37 (SD = 1.22). They also gave an average of $8.60 \mathrm{~km}$ for a usual run and/or 49.37 minutes per run. The women in this 
sample were well-educated $(82.9 \%$ with a university degree or higher) and all were employed outside of the home.

\subsection{Measures}

2.2.1. Positive Affect. The Positive Affect Negative Affect Schedule (PANAS) [37], comprised of 20 adjective words (10 positive, 10 negative), was used to assess participants' affect. According to the circumplex model, affect can be characterized by the dimensions of valence (positive, negative) as well as activation (low, high) and experts maintain that the PANAS focuses on the high activation component of affect [38]. For each adjective, participants rated their response from (1) not at all to (5) extremely using the stem Indicate to what extent you feel this way right now, that is, at the present moment. Examples of adjectives include: excited (positive) and upset (negative). Given the focus on positive affect in the present investigation, only these adjectives were summed and analyzed. In the present study, the Cronbach Alpha for the positive subscale pre- and post-running were .88 and .91 , respectively.

2.2.2. Motivation. The Situational Motivation Scale (SIMS) [39] was employed to measure situational motivation to run. The SIMS is comprised of 16 items across four subscales that assess the different behavioural regulations (intrinsic motivation, identified, external, and amotivation). Introjected regulation was excluded from the original SIMS in order to have more succinct instrument for research purposes [39]. In the current study, an enhanced version of the SIMS with four validated items tapping this type of regulation was used $[40,41]$. Items measuring introjection in the enhanced Situational Motivation Scale (SIMS) [41]: (1) because I would feel bad not doing it; (2) because I would feel guilty not to do it; (3) because I want to avoid feeling guilty; (4) because I would regret not doing it. Using a 7-point Likert Scale from (1) corresponds not at all to (7) corresponds exactly, participants respond to stem why are you currently [about to run], for items that included "Because I want to avoid feeling guilty" (introjection) and "Because I am doing it for my own good" (identified). Average score were calculated for intrinsic motivation, introjected regulation, and identified regulation. As expected from a sample of active participants, scores on external regulation and amotivation were low and variance levels were negligible, which contributed to low internal consistency values. No further analyses were conducted on these regulations. The Cronbach alphas of relevant subscales were acceptable to good with values of $.65, .85$, and .85 for identified regulation, introjected regulation and intrinsic motivation, respectively.

2.2.3. Perceived Intensity. The Rating of Perceived Exertion (RPE) Scale [42] was administered in the minutes immediately following the run to assess the perceived intensity/ exertion of the running activity. More specifically, and similar to previous studies we employed what some experts have coined "session RPE", whereby participants were instructed to rate the overall intensity of the full running session
$[43,44]$. This was expected to minimize the influence of momentary fluctuations in how participants' perceived their exertion, which could contaminate the accuracy of the overall evaluation. Numerical values from 6 to 20 make up the [session] RPE scale which is also anchored at every odd integer with a brief descriptor (i.e., 7 = very, very light; $13=$ somewhat hard; $19=$ very, very hard). The validity and reliability of the RPE are well established given its frequent usage in studies of PA and mood [45].

2.3. Procedures. This study was approved by the Ethics Review Board of the University of Ottawa and was part of a larger project. Interested subjects attended an individual session at a university laboratory space. Upon arrival, participants provided written informed consent and promptly responded to the SIMS and the PANAS prior to the running activity. Participants were taken to a private exercise room equipped with a treadmill, a desk, and a chair and were explained the running protocol. In order to mimic a selfpaced run and yet easily log the pace of the run to ensure that at least a moderate-intensity run was being met, the treadmill control panel was physically detached from the treadmill running belt. This allowed the researcher to easily adjust settings in response to any and all demands from the participant. The participants completed a 2-3 minute warm-up walk at an average speed of $5.17 \mathrm{~km} /$ hour. Next, the researcher sped up the belt to the participant's desired pace. The researcher remained in proximity in order to speed up or slow down the belt as frequently as necessary, the details and timing of which were duly noted. By weighting any change in pace by the ratio of elapsed time at that pace, an average running pace was computed. There was minimal conversation and eye contact between participant and researcher and the treadmill was maintained at a grade of zero. Participants ran for a 30-minute duration in order to stay consistent with previous inquiries on acute exercise and affective states (e.g., Bartholomew et al., 2005 [46]). Afterwards, the belt was slowed to the initial walking speed for a 2-minute cool-down and participants provided the session RPE for the 30-minute running component. Lastly, participants responded to the PANAS once again.

2.4. Analyses. All data were entered into SPSS Version 18.0; sums, means, and standard deviations were calculated. Initial data-screening procedures were conducted according to procedures outlined in Tabashnick and Fidell [47]. Namely, assessments were conducted for data entry errors, missing data, outliers, normality and the basic assumptions of regression analyses. Descriptive statistics and reliability analyses were calculated for affect and motivation variables. A repeated-measures $t$-test compared pre-run and post-run levels of positive affect as a preliminary examination of whether the run had a significant influence on participants' affect.

A standardized residual change score for positive affect was calculated for each participant in order to adequately account for participants' initial affect scores [48]. Namely, a predictor score was computed by regressing post-run affect on pre-run affect and then subtracting this from the observed 
TABLE 1: Means and standard deviations (SD) for situational motivational regulations, positive affect, and rating of perceived exertion (RPE).

\begin{tabular}{lcc}
\hline Variable & Mean & SD \\
\hline Intrinsic motivation for running & 5.80 & .82 \\
Identified regulation for running & 6.35 & .47 \\
Introjected regulation for running & 3.23 & 1.44 \\
Prerun positive affect & 33.39 & 6.36 \\
Postrun positive affect & 36.02 & 6.93 \\
RPE & 12.79 & 1.15 \\
\hline
\end{tabular}

scores. The residual change score served as the outcome variable in three separate hierarchical multiple-regression models that were employed to test the interaction between RPE and situational motivation for running. Specifically, separate product terms for RPE and the three motivational regulations were created using standardized scores and each term was added as the last step in their respective regression models.

\section{Results}

During the experimental running session, participants ran at an average pace of $9.66 \mathrm{~km} /$ hour and provided a mean RPE of 12.79. This value reflects a moderate to high intensity that was similar to the usual RPE achieved by the women outside of the laboratory. There were no univariate outliers on any of the variable. All assumptions regarding normality, linearity, and homoscedasticity were met and there was no evidence of collinearity. Positive affect increased significantly from pre- to post-run $[t(40)=4.83, P<.001]$. See Table 1 for descriptive statistics.

Results of the hierarchical regression analyses showed no significant interaction between intrinsic motivation and RPE on residual change scores in positive affect $\left[F_{\text {change }}\right.$ $(1,37)=.23, P=.63$ ] nor between identified regulation and RPE $\left[F_{\text {change }}(1,37)=1.82, P=.19\right]$. However, there was a significant interaction effect of RPE and introjection $\left[F_{\text {change }}(1,37)=4.20, \beta=-.30 P<.05\right]$ which explained an additional $9 \%$ of variance in the change in positive affect from the variables alone.

As displayed in Figure 1, when participants reported low introjection, the influence of perceived running intensity on the change in positive affect was considerable, with higher RPE being associated with a greater increase in affect from pre- to post-run. This effect became less pronounced with rising levels of introjection. That is, when participants reported high introjected regulation, the change in positive affect was elevated and fluctuated very little with rising RPE values, and even showed a trend toward diminishing slightly.

\section{Discussion}

The results of this experimental study revealed a significant interaction between RPE (i.e., intensity) and introjected regulation but not between RPE and intrinsic or identified
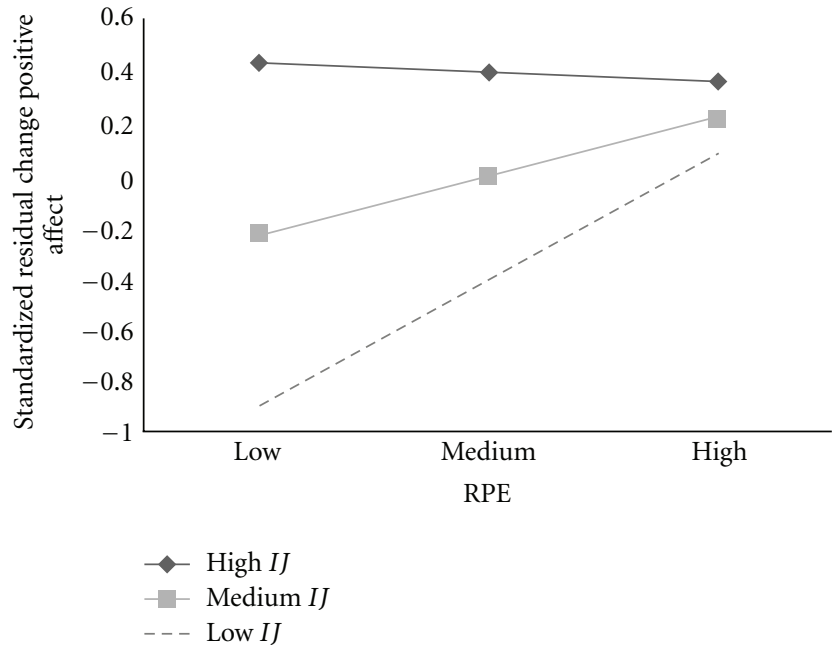

FIGURE 1: The interaction between introjected motivation for running (IJ) and Ratings of Perceived Exertion (RPE) on residualized change scores in positive affect.

styles of motivation in predicting changes in positive affect from pre- to post-running. To our knowledge, this is the first study to have examined this interplay, which builds on previous deliberations by Ekkekakis and Lind [49] regarding a potentially complex causal chain linking intensity, pleasure from exercise, and adherence, among other factors. In particular, our investigation offers an important adjunct to a study by Duncan and colleagues [20] that revealed associations between SDT's motivational regulations and PA intensity. Specifically, we considered the interaction between these variables in explaining a factor that is being increasingly recognized not only as an important consequence of PA but also as a viable determinant of future participation, namely positive affect [5]. Our results attest to recent suggestion that a person's motivational style for PA should be considered when attempting to maximize the affective gains of aerobic PA, especially at a self-selected intensity [16]. The strengths of this investigation include the self-paced nature of the running activity, a situational measure of motivation for running, and the use of a well-controlled laboratory environment.

Researchers have observed that up to very high exertion levels, there is a basic positive linear association between exercise intensity and affect [50]. While the basic linear relationship (RPE-positive affect) in this study was in fact positive for low and moderate levels introjection, it deviated markedly for those high in introjection, such that affective changes were fairly stable regardless of perceived intensity. While introjection has been associated with engaging in vigorous exercise [20], our results imply that this relationship may have little bearing on the acute mood changes that are experienced through PA, at least among avid female runners. This interplay may even be worrisome given a downward trend in positive affect as the RPE increased among highly introjected runners. This could suggest that active females that are high in introjection achieve some form of an immediate "feel-good" or "relief" effect from an activity such 
as running that serves to prevent or relieve feelings of guilt, thereby materializing irrespective of perceived intensity. This was evidenced in our study by greater overall gains in affect for those high in introjection, which may be akin to what Sabiston and colleagues [51] referred to as the reparative properties of motivation from guilt.

On the other hand, among runners lower in introjected regulation, changes in positive affect were associated with greater variability in RPE which could indicate a greater appreciation of the sensations and physical properties of PA. Lind et al. [52] remarked that individuals usually choose to exercise at a pace that improves or maintains their mood. Our findings show that this may be more applicable for exercisers with lower introjection and who are less driven by internal pressures to exercise, thereby freeing them to experience the PA session more fully. Therefore, in-task affective states and sensation, which were not assessed in our study, could be a source of discrepancy between persons high and low in introjection. Future studies should consider open-ended probes during acute PA sessions in order to ascertain the pertinent sources of affective changes between individuals that differ in motivational style.

Despite its effect on positive affect in our study, as well its influence on sustaining high intensity PA [20], introjection has been associated with several negative psychological consequences that were not assessed in our acute exercise study (e.g., lower self-worth and life satisfaction) [24]. Thus, cautious interpretation of our results is warranted as immediate improvements in positive affect may not necessarily translate into benefits in general well-being [51]. This could be disconcerting if we consider that individuals with lower levels of well-being are generally less likely to engage in PA [53], thus initiating a questionable cycle of regular PA maintenance and compounding issues related to leading a healthy and active lifestyle.

On a different note, the results showing nonsignificant interactions between more self-determined motivational styles and RPE may be of theoretical significance. Similar to what was discussed above with respect to runners with lower levels of introjection, our results hint that those with higher levels of intrinsic and/or identified regulation could have less contingencies attached to their exercise engagement. In this regard, Burton and colleagues [54] found that being intrinsically motivated positively predicted well-being independently of the level of performance. Moreover, they found that fostering intrinsic motivation may diminish certain contingencies between one's perceived performance and their wellbeing. This is consistent with SDT principles and research revealing that despite variability in perceived competence for an activity, self-determined individuals show greater interest, pleasure, and confidence which is exhibited through greater well-being [55]. Thus the relationship between selfdetermined regulations and well-being is more likely of a direct nature, as indicated in the present study whereby perceived intensity, which could be view as an indicator of their performance, did not significantly shape post-PA changes in positive affect among the women higher on self-determined regulations. Other authors have also noted a direct relationship between identified and intrinsic motivation and post-PA affect as an indicator well-being [22]. In addition, and from a psychometric perspective, ceiling effects could be partly to blame for nonsignificant findings given the high means and low variances for these variables, especially identified regulation.

With respect other possible limitations of this study, there are variant opinions in the literature regarding the optimal time point(s) at which to assess RPE. We opted to assess session RPE immediately post-PA which is common practice among researchers $[50,56]$. However, some authors such as Singh et al. [57] argue that post-PA RPE can vary significantly in the few minutes following exercise (e.g., between 5-10 minutes) and that evaluations of RPE taken 15-30 minutes after PA are more stable and valid indicators of participants' perceived intensity. Still, other experts gravitate away from session RPE altogether claiming that repetitive RPE measurements at regular intervals during a PA session provide a more representative measure of intensity $[58,59]$. In future studies, researchers will need to address concerns over the optimal time point(s) for RPE measurement(s) in studying exercise-related affect and they may wish to supplement such inquiries with alternative and objective measures of intensity. Similarly, researchers might wish to consider the use of research designs that capitalize on longitudinal and intask effects of motivation and intensity on indicators of wellbeing.

In addition, future studies will need to make use of larger samples and draw from groups of participants that are more diverse in terms of activity levels as this may alleviate some of the psychometric issues regarding the assessment of certain motivational styles. The use of only active, healthy-weight women could also be considered a drawback of the present study in terms of generalizability and it would be worthwhile to test the given interactions in overweight or obese individuals who may experience PA differently [60]. Researchers have already shown that autonomous regulations are associated with long-term weight management as well as indicators of well-being in obese populations $[23,61]$ and it would be worthwhile to examine the interplay with PA intensity in order to develop optimal interventions strategies for these individuals.

Yet it is also interesting to note that some studies have actually shown that affective experiences and pleasure from exercise might not significantly differ between normal weight and overweight women, at least at self-selected intensities $[29,49]$. In addition, other experts mention that it is actually fruitful to study an active population as much can be learned regarding the determinants of successful PA engagement and associated consequences and this could then be targeted among the insufficiently active [20]. Moreover, the purpose of this study was to explore a theory-based interaction mechanism and therefore it was advantageous to select a sample that could maximize the postulated relationships. In sum, while women higher in introjection showed the greatest change in positive affect post-running, further reasoning as to why this increase was not particularly sensitive to the self-selected intensity of the run (RPE), as well as the long-term impact of this relationship on well-being and PA maintenance, is left to future inquiries. 


\section{References}

[1] V. S. Conn, A. R. Hafdahl, and L. M. Brown, "Meta-analysis of quality-of-life outcomes from physical activity interventions," Nursing Research, vol. 58, no. 3, pp. 175-183, 2009.

[2] C. K. Martin, T. S. Church, A. M. Thompson, C. P. Earnest, and S. N. Blair, "Exercise dose and quality of life," Archives of Internal Medicine, vol. 169, no. 3, pp. 269-278, 2009.

[3] J. Reed, "Acute physical activity and self-reported affect: a review," in Causes, Roles, \& Influences of Mood States, A. V. Clark, Ed., pp. 91-113, Nova Science, Hauppauge, NY, USA, 2005.

[4] J. Reed and D. S. Ones, "The effect of acute aerobic exercise on positive activated affect: a meta-analysis," Psychology of Sport and Exercise, vol. 7, no. 5, pp. 477-514, 2006.

[5] D. M. Williams, S. Dunsiger, J. T. Ciccolo, B. A. Lewis, A. E. Albrecht, and B. H. Marcus, "Acute affective response to a moderate-intensity exercise stimulus predicts physical activity participation 6 and 12 months later," Psychology of Sport and Exercise, vol. 9, no. 3, pp. 231-245, 2008.

[6] B. M. Kwan and A. D. Bryan, "Affective response to exercise as a component of exercise motivation: attitudes, norms, selfefficacy, and temporal stability of intentions," Psychology of Sport and Exercise, vol. 11, no. 1, pp. 71-79, 2010.

[7] S. Lyubomirsky, L. King, and E. Diener, "The benefits of frequent positive affect: does happiness lead to success?" Psychological Bulletin, vol. 131, no. 6, pp. 803-855, 2005.

[8] P. J. O'Connor and T. W. Puetz, "Chronic physical activity and feelings of energy and fatigue," Medicine and Science in Sports and Exercise, vol. 37, no. 2, pp. 299-305, 2005.

[9] L. M. Van Landuyt, P. Ekkekakis, E. E. Hall, and S. J. Petruzzello, "Throwing the mountains into the lakes: on the perils of nomothetic conceptions of the exercise-affect relationship," Journal of Sport and Exercise Psychology, vol. 22, no. 3, pp. 208 234, 2000.

[10] J. Reed and S. Buck, "The effect of regular aerobic exercise on positive-activated affect: a meta-analysis," Psychology of Sport and Exercise, vol. 10, no. 6, pp. 581-594, 2009.

[11] P. Ekkekakis and S. J. Petruzzello, "Acute aerobic exercise and affect. Current status, problems and prospects regarding doseresponse," Sports Medicine, vol. 28, no. 5, pp. 337-374, 1999.

[12] S. Vazou-Ekkekakis and P. Ekkekakis, "Affective consequences of imposing the intensity of physical activity: does the loss of perceived autonomy matter," Hellenic Journal of Psychology, vol. 6, no. 2, pp. 125-144, 2009.

[13] D. M. Williams, "Exercise, affect, and adherence: an integrated model and a case for self-paced exercise," Journal of Sport and Exercise Psychology, vol. 30, no. 5, pp. 471-496, 2008.

[14] B. G. Berger and R. W. Motl, "Exercise and Mood: a selective review and synthesis of research employing the profile of mood states," Journal of Applied Sport Psychology, vol. 12, no. 1, pp. 69-92, 2000.

[15] S. J. H. Biddle and P. Ekkekakis, "Physically active lifestyles and well-being," in The Science of Well-Being, F. A. Huppert, B. Keverne, and N. Baylis, Eds., pp. 140-168, Oxford University, Oxford, UK, 2005.

[16] F. D. Legrand and J. Thatcher, "Acute mood responses to a 15minute long walking session at self-selected intensity: effects of an experimentally-induced telic or paratelic state," Emotion, vol. 11, no. 5, pp. 1040-1045, 2011.

[17] E. L. Deci and R. M. Ryan, Intrinsic Motivation and Self-Determination in Human Behaviour, Plenum, New York, NY, USA, 1985.

[18] E. L. Deci and R. M. Ryan, Handbook of Self-Determination Research, University of Rochester, Rochester, NY, USA, 2002.
[19] M. S. Hagger, N. L. D. Chatzisarantis, and J. Harris, "The process by which relative autonomous motivation affects intentional behavior: comparing effects across dieting and exercise behaviors," Motivation and Emotion, vol. 30, no. 4, pp. 307321, 2006.

[20] L. R. Duncan, C. R. Hall, P. M. Wilson, and O. Jenny, “Exercise motivation: a cross-sectional analysis examining its relationships with frequency, intensity, and duration of exercise," International Journal of Behavioral Nutrition and Physical Activity, vol. 7, no. 1, article 7, 2010.

[21] R. Puente and M. H. Anshel, "Exercisers' perceptions of their fitness instructor's interacting style, perceived competence, and autonomy as a function of self-determined regulation to exercise, enjoyment, affect, and exercise frequency," Scandinavian Journal of Psychology, vol. 51, no. 1, pp. 38-45, 2010.

[22] R. Lutz, M. Lochbaum, and K. Turnbow, "The role of relative autonomy in post-exercise affect responding," Journal of Sport Behavior, vol. 26, no. 2, pp. 137-155, 2003.

[23] P. N. Vieira, J. Mata, M. N. Silva et al., "Predictors of psychological well-being during behavioral obesity treatment in women," Journal of Obesity, vol. 2011, Article ID 936153, 8 pages, 2011.

[24] C. Thøgersen-Ntoumani and K. R. Fox, "Exploring the role of autonomy for exercise and its relationship with well-being: a study with non-academic University employees," International Journal of Sport \& Exercise Psychology, vol. 5, no. 3, pp. 227239, 2007.

[25] J. Edmunds, N. Ntoumanis, and J. L. Duda, "Examining exercise dependence symptomatology from a self-determination perspective," Journal of Health Psychology, vol. 11, no. 6, pp. 887-903, 2006.

[26] M. Gagné, R. M. Ryan, and K. Bargmann, “Autonomy support and need satisfaction in the motivation and well-being of gymnasts," Journal of Applied Sport Psychology, vol. 15, no. 4, pp. 372-390, 2003.

[27] E. L. Deci and R. M. Ryan, "The "what" and "why" of goal pursuits: human needs and the self-determination of behavior," Psychological Inquiry, vol. 11, no. 4, pp. 227-268, 2000.

[28] M. L. Schneider and D. J. Graham, "Personality, physical fitness, and affective response to exercise among adolescents," Medicine and Science in Sports and Exercise, vol. 41, no. 4, pp. 947-955, 2009.

[29] P. Ekkekakis, E. Lind, and S. Vazou, "Affective responses to increasing levels of exercise intensity in normal-weight, overweight, and obese middle-aged women," Obesity, vol. 18, no. 1, pp. 79-85, 2010.

[30] M. D. Hoffman and D. R. Hoffman, "Exercisers achieve greater acute exercise-induced mood enhancement than nonexercisers," Archives of Physical Medicine and Rehabilitation, vol. 89, no. 2, pp. 358-363, 2008.

[31] S. M. Markowitz and S. M. Arent, "The Exercise and affect relationship: evidence for the dual-mode model and a modified opponent process theory," Journal of Sport and Exercise Psychology, vol. 32, no. 5, pp. 711-730, 2010.

[32] K. S. Kelsey, B. M. DeVellis, M. Begum, L. Belton, E. G. Hooten, and M. K. Campbell, "Positive affect, exercise and self-reported health in blue-collar women," American Journal of Health Behavior, vol. 30, no. 2, pp. 199-207, 2006.

[33] M. Kull, "The relationships between physical activity, health status and psychological well-being of fertility-aged women," Scandinavian Journal of Medicine and Science in Sports, vol. 12, no. 4, pp. 241-247, 2002.

[34] M. L. Segar, J. S. Eccles, and C. R. Richardson, "Type of physical activity goal influences participation in healthy midlife 
women," Women's Health Issues, vol. 18, no. 4, pp. 281-291, 2008.

[35] G. Godin and R. J. Shephard, "A simple method to assess exercise behavior in the community.," Canadian Journal of Applied Sport Sciences, vol. 10, no. 3, pp. 141-146, 1985.

[36] J. H. Kerr and G. Kuk, "The effects of low and high intensity exercise on emotions, stress and effort," Psychology of Sport and Exercise, vol. 2, no. 3, pp. 173-186, 2001.

[37] D. Watson, L. A. Clark, and A. Tellegen, "Development and validation of brief measures of positive and negative affect: the PANAS scales," Journal of Personality and Social Psychology, vol. 54, no. 6, pp. 1063-1070, 1988.

[38] P. Ekkekakis and S. J. Petruzzello, "Analysis of the affect measurement conundrum in exercise psychology: IV. A conceptual case for the affect circumplex," Psychology of Sport and Exercise, vol. 3, no. 1, pp. 35-63, 2002.

[39] F. Guay, R. J. Vallerand, and C. Blanchard, "On the assessment of situational intrinsic and extrinsic motivation: the Situational Motivation Scale (SIMS)," Motivation and Emotion, vol. 24, no. 3, pp. 175-213, 2000.

[40] M. Standage, D. C. Treasure, J. L. Duda, and K. A. Prusak, "Validity, reliability, and invariance of the Situational Motivation Scale (SIMS) across diverse physical activity contexts," Journal of Sport and Exercise Psychology, vol. 25, no. 1, pp. 1943, 2003.

[41] N. Gillet, R. J. Vallerand, M. A. K. Lafreniere, and J. S. Bureau, "The mediating role of positive and negative affect in the situational motivation-performance relationship: a test of the Self-Determination and broaden-and-build theories," In press.

[42] G. A. V. Borg, "Psychophysical bases of perceived exertion," Medicine and Science in Sports and Exercise, vol. 14, no. 5, pp. 377-381, 1982.

[43] C. Foster, J. A. Florhaug, J. Franklin et al., "A new approach to monitoring exercise training," Journal of Strength and Conditioning Research, vol. 15, no. 1, pp. 109-115, 2001.

[44] T. W. Sweet, C. Foster, M. R. McGuigan, and G. Brice, "Quantitation of resistance training using the session rating of perceived exertion method," Journal of Strength and Conditioning Research, vol. 18, no. 4, pp. 796-802, 2004.

[45] W. D. Russell and M. Newton, "Short-term psychological effects of interactive video game technology exercise on mood and attention," Educational Technology and Society, vol. 11, no. 2, pp. 294-308, 2008.

[46] J. B. Bartholomew, D. Morrison, and J. T. Ciccolo, "Effects of acute exercise on mood and well-being in patients with major depressive disorder," Medicine and Science in Sports and Exercise, vol. 37, no. 12, pp. 2032-2037, 2005.

[47] B. G. Tabachnick and L. S. Fidell, Using Multivariate Statistics, Allyn \& Bacon, Boston, Mass, USA, 5th edition, 2007.

[48] Y. D. Miller, S. G. Trost, and W. J. Brown, "Mediators of physical activity behavior change among women with young children," American Journal of Preventive Medicine, vol. 23, no. 2, pp. 98-103, 2002.

[49] P. Ekkekakis and E. Lind, "Exercise does not feel the same when you are overweight: the impact of self-selected and imposed intensity on affect and exertion," International Journal of Obesity, vol. 30, no. 4, pp. 652-660, 2006.

[50] C. A. Rocheleau, G. D. Webster, A. Bryan, and J. Frazier, "Moderators of the relationship between exercise and mood changes: gender, exertion level, and workout duration," Psychology and Health, vol. 19, no. 4, pp. 491-506, 2004.

[51] C. M. Sabiston, J. Brunet, K. C. Kowalski, P. M. Wilson, D. E. Mack, and P. R. E. Crocker, "The role of body-related self-conscious emotions in motivating women's physical activity," Journal of Sport and Exercise Psychology, vol. 32, no. 4, pp. 417-437, 2010.

[52] E. Lind, R. R. Joens-Matre, and P. Ekkekakis, "What intensity of physical activity do previously sedentary middle-aged women select? Evidence of a coherent pattern from physiological, perceptual, and affective markers," Preventive Medicine, vol. 40, no. 4, pp. 407-419, 2005.

[53] B. Roshanaei-Moghaddam, W. J. Katon, and J. Russo, "The longitudinal effects of depression on physical activity," General Hospital Psychiatry, vol. 31, no. 4, pp. 306-315, 2009.

[54] K. D. Burton, J. E. Lydon, D. U. D’Alessandro, and R. Koestner, "The differential effects of intrinsic and identified motivation on well-being and performance: prospective, experimental, and implicit approaches to self-determination theory," Journal of Personality and Social Psychology, vol. 91, no. 4, pp. 750-762, 2006.

[55] R. M. Ryan and E. L. Deci, "Self-determination theory and the facilitation of intrinsic motivation, social development, and well-being," American Psychologist, vol. 55, no. 1, pp. 68-78, 2000.

[56] N. Du, S. Bai, K. Oguri et al., "Heart rate recovery after exercise and neural regulation of heart rate variability in 30-40 year old female marathon runners," Journal of Sports Science and Medicine, vol. 4, no. 1, pp. 9-17, 2005.

[57] F. Singh, C. Foster, D. Tod, and M. R. McGuigan, "Monitoring different types of resistance training using session rating of perceived exertion," International Journal of Sports Physiology and Performance, vol. 2, no. 1, pp. 34-45, 2007.

[58] K. A. M. Ginis, M. E. Jung, and L. Gauvin, "To see or not to see: effects of exercising in mirrored environments on sedentary women's feeling states and self-efficacy," Health Psychology, vol. 22, no. 4, pp. 354-361, 2003.

[59] B. M. Miller, J. B. Bartholomew, and B. A. Springer, "Postexercise affect: the effect of mode preference," Journal of Applied Sport Psychology, vol. 17, no. 4, pp. 263-272, 2005.

[60] C. M. Sabiston, M. H. McDonough Meghan, W. A. Sedgwick Whitney, and P. R. E. Crocker Peter, "Muscle gains and emotional strains: conflicting experiences of change among overweight women participating in an exercise intervention program," Qualitative Health Research, vol. 19, no. 4, pp. 466-480, 2009.

[61] M. N. Silva, D. Markland, E. V. Carraça et al., "Exercise autonomous motivation predicts 3-yr weight loss in women," Medicine and Science in Sports and Exercise, vol. 43, no. 4, pp. 728-737, 2011. 


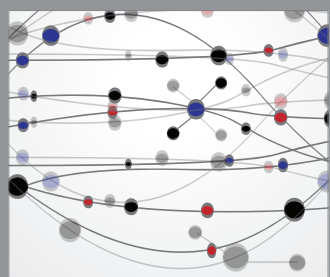

The Scientific World Journal
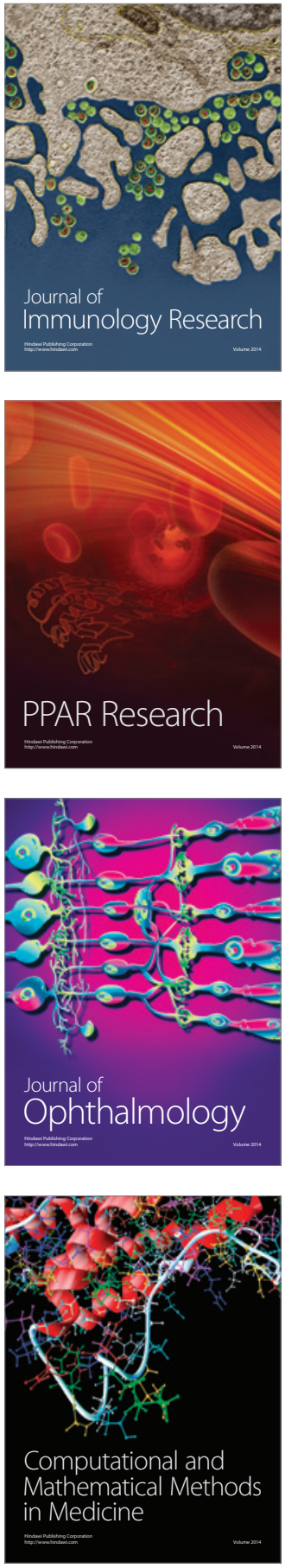

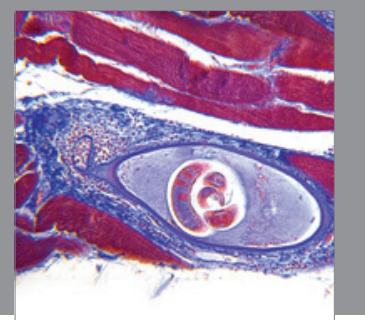

Gastroenterology

Research and Practice
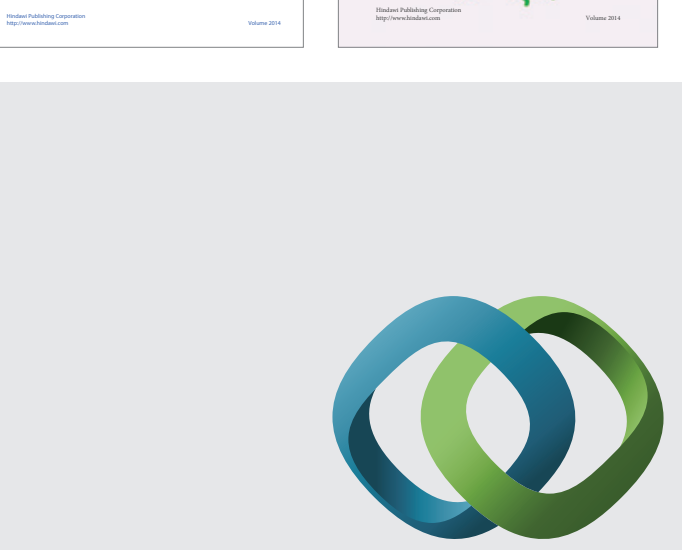

\section{Hindawi}

Submit your manuscripts at

http://www.hindawi.com
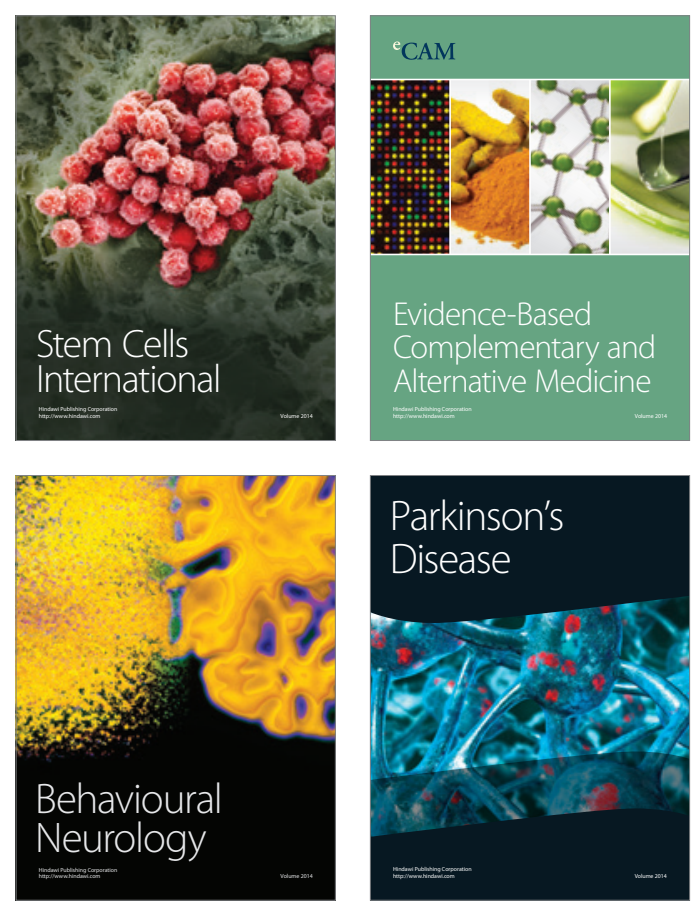

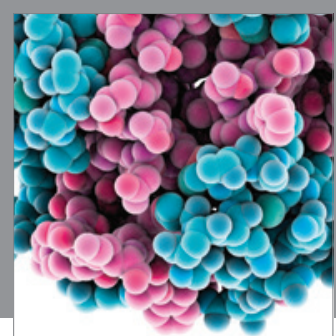

Journal of
Diabetes Research

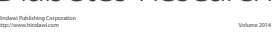

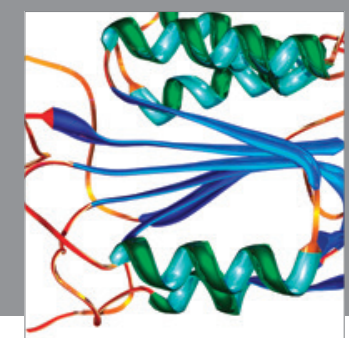

Disease Markers
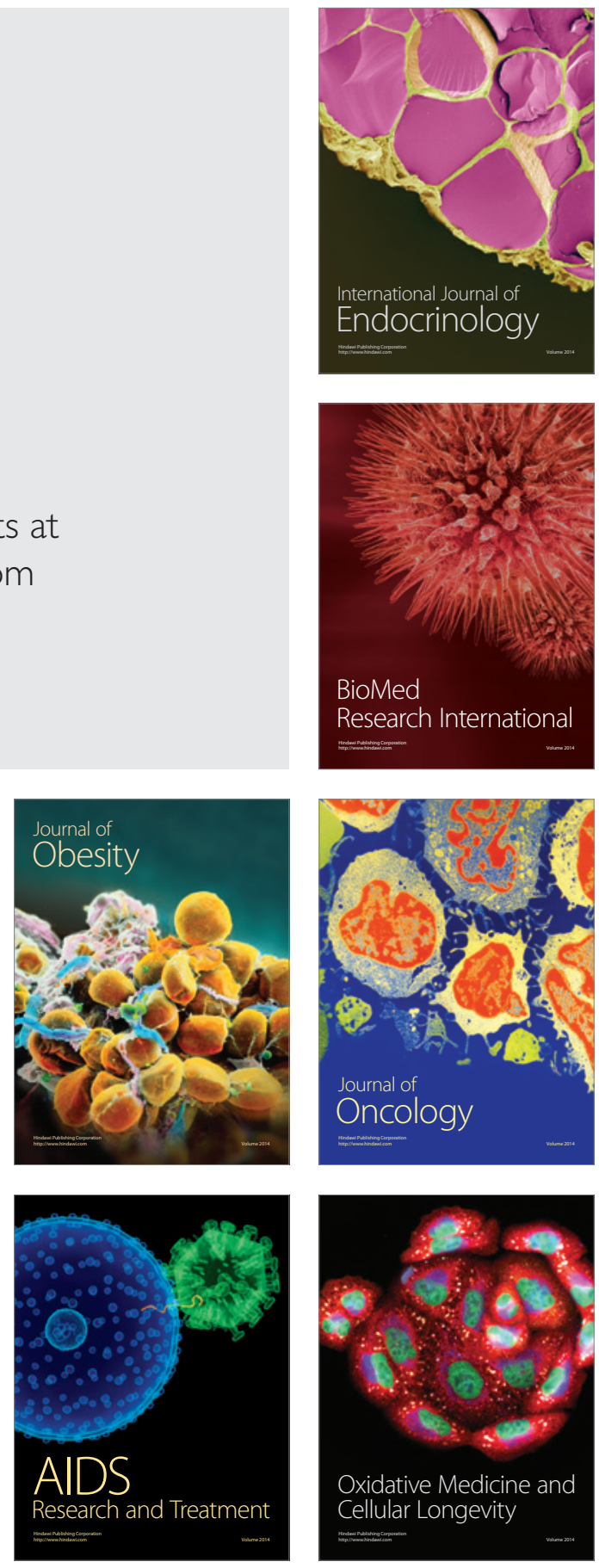